\title{
Recent Research Trends in Fabrication and Applications of Plant Essential Oil Based Nanoemulsions
}

Joyce Nirmala $\mathbf{M}^{*}$ and Nagarajan $\mathbf{R}$

Department of Chemical Engineering, Indian Institute of Technology Madras, Chennai-600 036, India

\begin{abstract}
This review encompasses on the most recent applications and fabrication methods of plant essential oil based nanoemulsions. The spice based nanoemulsions have promising medicinal applications like antimicrobial and anticancer activity.
\end{abstract}

Keywords: Nanoemulsions; Antimicrobial; Fabrication

\section{Introduction}

\section{Emulsions}

The definition of emulsion continued since 1930's. According to International Union of Pure and Applied Chemistry (IUPAC), emulsion is stated as "In an emulsion, liquid droplets and/or liquid crystals are dispersed in a liquid" [1]. The term emulsion was derived from the word emulgeo (meaning "to milk"). Milk is the best example for natural emulsion. Emulsions could be water-in-oil (W/O), oil-in-water $(\mathrm{O} / \mathrm{W})$ and oil-in-oil $(\mathrm{O} / \mathrm{O})$ type. Emulsifier acts as a third component playing an important role in dispersing these two immiscible liquids (dispersed phase and continuous phase). The sizes of globules formed are of 0.25 25 micrometers in diameter. Emulsions, the heterogenous system are classified based on the nature of emulsifier used and structure of the system formed. Macroemulsions, nanoemulsions, microemulsions or micellar emulsions, mixed emulsions, double and multiple emulsions are the various structures of the system. The major critical factor concerning emulsion is their breakdown processes. Creaming, sedimentation, flocculation, Ostwald ripening, coalescence and phase inversion are the various breakdown processes involved in causing instability of the emulsion formed. Oxidation, microbial contamination and adverse storage conditions are some of the types of chemical instability. Emulsions are evaluated by various methods such as dye test, dilution test, conductivity, fluorescence test, accelerated stability, globule size analysis and macroscopic examination.

Emulsions have wide applications in everyday life and they have a long history. The concept of emulsions is widely applied in many industrial sectors. The various applications of emulsions are food products (dairy, bakery products, candy products, meat products and beverages), agrochemicals (self-emulsifiable oils, emulsion concentrates and pesticides), oil (oil slick dipersions and petroleum based), paint (alkyd resin based products and latex emulsions), pharmaceutical (creams, ointments, anaesthetics, chemotherapy), cosmetics (hair and skin conditioning products), personal care (creams, lotions), dry cleaning formulations, Bitumen emulsions (road construction techniques) and others [2-6].

\section{Nanoemulsions}

Nanoemulsions are explored with great importance in recent decades due to their unique properties such as robust stability, transparent nature, high surface area and tunable rheology. Nanoemulsions have droplets of the order of $50 \mathrm{~nm}$. The common methods involved in nanoemulsion preparation include high energy methods (high pressure homogenization and ultrasonication) and low energy methods (phase inversion temperature and emulsion inversion point). Owing to their exceptional properties, nanoemulsions are widely used in drug delivery systems, food, cosmetics and pharmaceutical industries.

Nanoemulsions are colloidal nanodispersions of oil and water being thermodynamically stabilized by interfacial layer of surfactant/ co-surfactant. The surfactants are chosen from 'Generally Regarded as Safe' category from FDA for use in food and pharmaceutical preparations. Nanoemulsions are better preferred due to higher solubilization capacity and thermodynamic stability as compared to unstable emulsions and suspensions. The enhanced surface area and free energy makes it a unique transport system as well. The major components of nanoemulsions are oil phase, surfactant/cosurfactant/co-solvent and aqueous phase. Oils in nature are comprised of triglyceride mixtures that contain varying chain length or degrees of unsaturation of fatty acids. Triglycerides may also be synthetically hydrogenated to reduce oxidative degradation. The selection of oil component is crucial as it enhances solubilization potential of a drug candidate, and thereby, enables high drug loading capacity as well. Surfactants of HLB value less than 10 are hydrophobic (eg. Sorbitan monoesters and tends the formation of water-in-oil type. Surfactants with HLB value greater than 10 are hydrophilic (polysorbate 80 ), and tends formation of oil-in-water type. Sometimes, mixtures of both hydrophilic and hydrophobic surfactants are used for nanoemulsion formation. Ionic surfactants are not generally preferred as compared to non-ionic surfactants due to toxicological limitations. It is quite important in determining the surfactant concentration as increased amounts could cause gastrointestinal irritation. Lecithins, polaxamer, polysorbates are some of the most preferred surfactants for use. It should be noted that surfactants play a major role in reducing droplet size diameter. Co-surfactant, used on the other hand, also helps in reducing the interfacial tension between the oil-water interfaces. These co-surfactants penetrate the monolayer of surfactant causing more fluidity to interfacial film and breaks down the rigid surfactant film

*Corresponding author: Joyce Nirmala M, Department of Chemical Engineering Indian Institute of Technology Madras, Chennai, 600 036, India, Tel: 9677174200 ; E-mail: joycegitz@gmail.com

Received: February 15, 2017; Accepted: March 24, 2017; Published: March 31 2017

Citation: Nirmala MJ, Nagarajan R (2017) Recent Research Trends in Fabrication and Applications of Plant Essential Oil Based Nanoemulsions. J Nanomed Nanotechnol 8: 434. doi: 10.4172/2157-7439.1000434

Copyright: @ 2017 Nirmala MJ, et al. This is an open-access article distributed under the terms of the Creative Commons Attribution License, which permits unrestricted use, distribution, and reproduction in any medium, provided the original author and source are credited. 
formed by disrupting their liquid crystalline phases. Co-surfactants could not form self-associated structures like surfactants do. Organic solvents such as propylene glycol, polyethylene glycol, ethanol, glycerol, etc., are also generally preferred. They help in the dissolution of drug in lipid base or hydrophilic surfactants in large amounts by their nature of co-solvency and reduce dielectric constant of water. The $\mathrm{pH}$ and ionic nature of aqueous phase are given importance as they also influences droplet size and stability of nanoemulsions formed $[7,8]$.

Nanoemulsion formulation: The major components involved in the nanoemulsion formulation could be explained as follows.

The Oil phase: The physico-chemical characteristics of the oil phase determine the formation, stability and properties of a nanoemulsion. The bulk physico-chemical properties of the oil phase may be polarity, water solubility, viscosity, interfacial tension, density, refractive index, phase behaviour and its chemical stability. The oil phase is usually selected from non-polar components like triacylglycerols, diacylglycerols, monacylglycerols, free fatty acids, essential oils, mineral oils, flavouring oils, waxes, fat substitutes and others.

The Aqueous phase: The aqueous phase used in the nanoemulsion formulation is usually water, and also, other polar components. The polar compounds may include co-solvents, carbohydrates, proteins, minerals, acids and bases. The concentration of these components used and their type determines the $\mathrm{pH}$, ionic strength, polarity, density, rheology, refractive index, interfacial tension and phase behaviour of the aqueous phase, and this have a direct impact on the formation, stability and physicochemical characteristics of the nanoemulsion formed.

Stabilizers: To overcome the nanoemulsion breakdown processes such as flocculation, coalescence, Ostwald ripening and gravitational separation, different kinds of stabilizers are used to enhance their stability.

Emulsifiers: An emulsifier is capable of adsorbing to the droplet surfaces, promoting droplet disruption, and prevents droplets against the formation of aggregates. Hence, the selection of this surfaceactive molecule plays a vital role. In the case of high-energy methods, emulsifier causes droplet disruption by reducing the interfacial tension, and thereby, yields small droplets. In the case of low-energy methods, the ability of emulsifier to produce low interfacial tension leads to spontaneous formation of small droplets at certain environmental and solutions conditions. The small molecules are classified as ionic, nonionic and zwitterionic based on the electrical characteristics [9-15].

Fabrication techniques: Nanoemulsions are non-equilibrium systems and their preparation involves large amount of either energy or surfactants or combinations of both. Methods of preparation of nanoemulsion involve high energy methods (high-pressure homogenizer, microfluidizer and ultrasonicator) and low energy methods (self-emulsification, phase transition and phase inversion methods)

The high-energy method utilizes mechanical devices to produce strong disruptive forces to break the oil and water phase to obtain nanoemulsions. The low-energy method utilizes the stored internal energy for the formation of small droplets. Emulsions are obtained by changing the process parameters like temperature, composition and others that affect the hydrophilic-lipophilic balance (HLB).

Nanoemulsions require higher energy for its production as compared to macroemulsions. Thus, surfactants play a main role in lowering the surface tension between the oil-water interfaces. Non- ionic surfactants reduce surface tension to a greater extent as compared to polymeric surfactants. The effect of surfactant on the interfacial dilatational modulus is also important. Surfactants also prevent shear induced coalescence during emulsion process.

High pressure homogenization: The use of high-pressure homogenizer or piston homogenizer produces nanoemulsions of very small droplet size of up to $1 \mathrm{~nm}$ range. Hydraulic shear, intense turbulence and cavitational forces act together to yield smaller droplets. We could resubject the resultant product with homogenizer until a desired droplet size and polydispersity index is obtained. Emulsions are prepared at high volume faction of the dispersed phase and diluted later. But, this could result in coalescence during the emulsification process, and for this purpose, surfactant that causes effective reduction in surface tension is preferred.

Microfluidization: This is a patented technology. Microfluidizer uses a high-pressure positive displacement pump (500 - 20,000 psi). This forces the product through the interaction chamber that consists of small micro-channels to an impingement area, and thus, results in fine particles of submicron range. Initially, the aqueous phase and oily phase together are processed in an inline homogenizer to obtain coarse emulsion. This is then forced into a microfluidizer for further processing to yield a nanoemulsion formulation. This process of introducing coarse emulsion into micro-channels of microfluidizer is repeated many times till a desired particle size is attained. The emulsion obtained is filtered under nitrogen to eliminate any undesired larger droplets present. Thus, a stable and uniform nanoemulsion is formed.

Both high-pressure homogenization and microfluidization techniques could be used for nanoemulsion preparation at laboratory and industrial scale.

Ultrasonication: In ultrasonic emulsification, strong shear forces are produced by ultrasonic cavitation that produces bubbles and breaks down the particle size to nanometric range. Ultrasonication technique is employed in small scale production and mainly used at laboratory scale. This method is used as an alternative for high pressure homogenization. The method utilizes reduced surfactant concentration and low energy to yield homogenous nanoemulsion, and makes it more advantageous than conventional mechanical processes.

Phase inversion temperature: The phase inversion temperature method demonstrates a relationship between minimum droplet size and complete solubilization of oil in a bicontinuous phase that is independent of the initial phase equilibrium (single or multiphase). Phase inversion in emulsions could be of two types: transitional inversion and catastrophic inversion. The former is induced by changing factors like temperature or electrolyte concentration that affect the HLB of the system. The latter is induced by changing the HLB number of the surfactant at constant temperature.

Phase inversion temperature method employs temperaturedependent solubility of non-ionic surfactants to change their affinities for water and oil as a function of temperature. In this method, oil, water and non-ionic surfactants are mixed together at room temperature. This mixture consists of oil-in-water microemulsions with excess oil, and the surfactant monolayer exhibits positive curvature. When this formation is heated gradually, the surfactant (polyethoxylated) becomes lipophilic due to dehydration of polyoxyethylene groups, and this forms the basis of nanoemulsion formation. At higher temperatures, this surfactant gets fully solubilized in the oily phase. Thus, a phase inversion from oil-in-water to water-in-oil macroemulsion occurs and the surfactant monolayer demonstrates a negative curvature at this point. This 
method is not preferred for the incorporation of thermolabile drugs due to heating of components involved.

Nanoemulsion generally shows stability against sedimentation or creaming factors. But, Ostwald ripening remains a main concern for breakdown processes of nanoemulsion formation.

Phase inversion composition (Self-nanoemulsification): The phase inversion composition has drawn great attention as nanoemulsions could be formed at room temperature without the use of heat energy or organic solvents. They produce small droplet size of approximately $50 \mathrm{~nm}$ and are highly kinetically stable, though, they are not thermodynamically stable. A stepwise addition of water into solution of surfactant in oil with gentle stirring at constant temperature yield nanoemulsions. The spontaneous nanoemulsification technique relates to the phase transitions and involves lamellar liquid crystalline phases or D-type bicontinuous microemulsion during the emulsification process.

Solvent displacement: The solvent displacement method is developed nano-precipitation technique used for the preparation of polymeric nanoparticles. In the solvent displacement technique, the oily phase is dissolved in organic solvents (acetone, ethanol and ethyl methyl ketone). The organic phase is added to an aqueous phase containing surfactant to produce spontaneous nanoemulsion by rapid diffusion of organic solvent. The organic solvents used could be removed by vacuum evaporation or other suitable methods. Sometimes, nanoemulsion could be formed even without the use of surfactant. This method could be done at room temperature with simple stirring, and hence, used for parenteral formulations. One of the major limitations of this method is the high ratio of solvent used to yield a desired droplets range. Moreover, the process of solvent removal requires additional input and is tedious during scale-up processes [16-23].

Stability: Nanoemulsions possess enhanced physical and chemical stability of drugs. Stability is one of the major concerns regarding the development of emulsions, microemulsions and nanoemulsions. Stability studies are performed by storing the samples in both refrigerated conditions and room temperature over a period of months. Stability is determined with respect to insignificant changes in viscosity, refractive index and droplet size during storage conditions. Accelerated stability studies are also performed for nanoemulsion formulations at accelerated temperatures. The drug content is examined by HPLC technique to determine stability of the formulated nanoemulsion.

Though, nanoemulsions appear physically similar as microemulsions, they have a critical difference. Nanoemulsions are kinetically stable, whereas, microemulsions are thermodynamically stable. Nanoemulsions could be formed be mechanical shear and microemulsions are formed through self-assembly. Moreover, nanoemulsions have very small droplet size and possess higher stability against sedimentation or creaming [24-28].

Characterization of nanoemulsion: To identify the structure and behaviour of nanoemulsions, more sophisticated instrumentation such as dynamic light scattering, $\mathrm{x}$-ray or neutron scattering, atomic force microscopy, cryo-electron microscopy may be required. Various parameters involved in assessing nanoemulsions are discussed as follows.

Morphology: The morphology of nanoemulsions is determined by scanning electron microscopy and (SEM) and transmission electron microscopy (TEM). In SEM, the samples are examined at an accelerating voltage of about $20 \mathrm{kV}$ at various magnifications to obtain the surface morphology of the dispersed phase. A three-dimensional image of the globules or particles is determined using SEM.

A higher resolution image of the dispersed phase is obtained through TEM. In TEM, the sample is initially negatively stained with $1 \%$ aqueous solution of phosphotungstic acid or by dropping $2 \%$ uranyl acetate solution to a $200 \mu \mathrm{m}$ mesh size Pioloform ${ }^{\mathrm{Tm}}$-coated copper grid or a microscopic carbon-coated grid. This is then observed under TEM at appropriate voltage. A digital image processing programme could be used for qualitative measurements size and its distribution. More sophisticated instrumentations like x-ray or neutron scattering, atomic force microscopy and cryo-electron microscopy are also preferred to exploit the structure and behaviour of nanoemulsions.

Droplet size and zeta potential: Dynamic light scattering (DLS) or the photon correlation spectroscopy (PCS) measures the fluctuations in the intensity of scattering by droplets or particles due to Brownian motion. Nanoemulsion droplet size, polydispersity and zeta potential can be assessed by PCS using a particle size analyzer. The polydispersity index is a measure of the broadness of the size distribution obtained from the cumulative analysis of dynamic light scattering technique. The polydispersity index determines homogeneity of the emulsion dispersion. PCS gives z-average particle diameter. Laser diffraction is yet another technique used for measuring particle size.

Viscosity measurement: Viscosity is an important criterion for assessing stability and efficient drug release. It is measured using a viscometer. A Brookfield type rotary viscometer measures viscosity at different shear rates at various temperatures. Viscosity of nanoemulsions is a function of surfactant, water and oil components and their concentrations.

Conductivity meter: To determine the type $(\mathrm{O} / \mathrm{W}$ or $\mathrm{W} / \mathrm{O})$ of nanoemulsion formed, the phase system of nanoemulsions is determined by electrical conductivity. In case of $\mathrm{O} / \mathrm{W}$ type, the external phase is water; hence, they are highly conducting. In case of $\mathrm{W} / \mathrm{O}$ type, the internal phase is water; hence, non conducting. In certain cases of W/O type, there is a sharp rise in conductivity at low volume fractions, and this percolative behaviour occurs before the formation of bicontinuous structures. The electrical conductivity measurements are helpful in determining the nature of continuous phase and phase inversion phenomena.

$p H$ and refractive index: The apparent $\mathrm{pH}$ of the formulation is determined by $\mathrm{pH}$ meter. The refractive index was measured using refractometer.

Dye solubilisation: An oil soluble dye is solubilised within the oil phase of $\mathrm{O} / \mathrm{W}$ and is dispersible in the W/O globule. A water soluble dye is solubilised within the aqueous phase of $\mathrm{W} / \mathrm{O}$ and is dispersible in the $\mathrm{O} / \mathrm{W}$ globule.

Dilutability test: The O/W type nanoemulsion is dilutable with water, but the W/O type is not dilutable and undergoes phase inversion into $\mathrm{O} / \mathrm{W}$ type.

Stability: Various stress tests are performed to confer stability of the formed emulsion. Centrifugation is done at $3500 \mathrm{rpm}$ initially to check physical stability. Followed by, heating-cooling cycle at $4^{\circ} \mathrm{C}$ and $45^{\circ} \mathrm{C}$ for $48 \mathrm{~h}$ each. This cycle is repeated for a period of six cycles. The preparations that did not phase separate is further taken for freeze thaw cycle. Here, the samples are subjected to $-21^{\circ} \mathrm{C}$ and $+25^{\circ} \mathrm{C}$ for $48 \mathrm{~h}$ each. This is repeated for a period of three cycles to determine stability.

In Vitro skin permeation studies: The drug release profile of nanoemulsions could be measured using Franz diffusion cell. The drug 
release is studied by dispersing a known amount of the formulation in the donor compartment of a Franz cell containing a membrane barrier, and then monitoring the appearance of the encapsulated drug in the receptor compartment (containing phosphate buffered saline, $\mathrm{pH} 7.4$; $100 \mathrm{rpm} ; 37 \pm 1^{\circ} \mathrm{C}$ ). About $1 \mathrm{ml}$ of the sample is withdrawn from the receptor medium at regular intervals and is replaced with an equal amount of medium. This withdrawn sample is then filtered using $0.22-$ $50 \mu \mathrm{m}$ filter and the drug released is analyzed using HPLC or UV-Vis spectroscopy at wavelength of peak absorption of the drug.

In Vivo bioavailability studies: In the in vivo bioavailability or the pharmacodynamic studies, the formulation/preparation is applied to whole live animal. The blood samples are withdrawn at definite intervals and then centrifuged. The plasma is then analysed for drug content using HPLC. Both the results of in vitro and in vivo studies demonstrate the bioavailability of the drug preparation. The pharmacodynamic properties of the formulations are determined depending on the pharmacological properties of the incorporated drug $[29,30]$.

Applications: The research on nanoemulsions for its potential applications in various fields has been pondered for a decade of years. The expectations of nanoemulsions on direct applications as final products are not many, owing to stability as limitation factor. The Ostwald ripening mechanism which breaks the small droplet size of the formed nanoemulsions plays a critical reason. On the other hand, nanoemulsions using silicone oils as dispersed phase has also shown great stability but their application is still not developed. In some cases, the applications of nanoemulsions is quite effective if the nanoemulsion formulations is prepared shortly just before use for applications. Here, the droplet size diameter might increase with respect to increase in time and could not be used for usage in applications. Though, the direct applications of nanoemulsions in food technology and agrochemicals have not been fully exploited, the pharmaceutical field has been greatly benefited.

Self emulsifying nanoemulsion systems for parenteral and oral applications have been demonstrated for its potential use. The stability problem is overcome by using these systems soon after their preparation. The development of nanocarriers has helped in the treatment of many diseases. Some of the anti-convulsant, anti-hypertensive, antibiotic and anti-inflammatory drugs have been solubilised in nanoemulsions for use. Also, drugs solubilised in nanoemulsions are been investigated for cancer therapy and HIV/AIDS therapy. Various other investigations on nanoemulsions such as applications as anthrax vaccine, inactivation of Ebola virus, anti-microbial generic activity, efficacy of schistosomicidal compound, intestinal absorption of three model drugs and others are being carried out [31]. Nanoemulsions of ramipril (antihypertensive drug), celecoxib (arthritis and osteoarthritic treatment), carbamazepine (anticonvulsant drug) and calixarene entrapped nanoemulsion for uranium skin decontamination have also been investigated. Nanoemulsions for treating coronary artery diseases, malaria, delivery of plasmid DNA and ocular drug delivery are also studied.

Nanoemulsions enable targeted delivery and hence made a revolution in the targeted treatment of many cancer diseases. This enhances the effectiveness of cancer therapeutic agents by eliminating tumors without affecting the healthy tissues. They enhance site specificity, withstand multidrug resistance and efficiently delivers therapeutic agent. The expression of folate receptors are been explored to target therapeutic compounds directly to cancer cells. Though, nanoemulsions are being explored for cancer treatment, prevention and dectection, their exact dosages and long term effects on cancer therapy is yet to be studied [32].
Recently, a new technological advancement of theranostic nanomedicine is considered promising towards personalized medicine. Of this accord, a celecoxib loaded near-infrared labelled perfluorocarbon nanoemulsion on three levels of scales was produced by microfluidization technique. There was no significant result of nanoemulsion scales on cell toxicity and pharmacological effects [33].

Some of the examples of nanocosmeceuticals are creams and lotions formulated with Buriti oil, palm oil esters-in-water and virgin coconut oil-in-water.

A new method was developed to produce six nano disperse dyes using $\mathrm{O} / \mathrm{W}$ nanoemulsions of $110-130 \mathrm{~nm}$ in range using microfluidization technique. A novel method produced rod shaped $2 \mathrm{Li}_{2} \mathrm{O} / \mathrm{MgO}$ catalyst nanoparticles on the inner surface of porous carriers $\left(\alpha-\mathrm{Al}_{2} \mathrm{O}_{3}\right)$ for oxidative coupling of methane reaction. This W/O nanoemulsion was produced by ultrasonic probe [34].

Nanoemulsions are used in combustion process of fuels to improve engine efficiency. A water-in diesel nanoemulsion was formulated by blending different proportions of water with nano-Al additives to improve the combustion characteristics. Triton X-100 was the surfactant used. The emulsion formed was of the nanometer range and the zeta potential values were neutral at water-diesel interface. Moreover, the blend was thermodynamically stable. The authors imply that the formulated nanoemulsion fuels has to be further tested for its fuel properties and engine performance to ascertain their potent application as future sustainable fuel alternative [35]

Thus, the recent trends on the direct applications of nanoemulsions are found more pronounced in the field of pharmaceutics and cosmetics.

\section{Essential Oils - An Overview}

A $16^{\text {th }}$ century Swiss reformer of medicine, Paracelsus von Hohenheim named an effective component of a drug as Quinta essentia. Thus, the term essential oil was thought to be originally derived. The "green" consumerism has led to scientific exploration in these compounds.

Till date, there are thousands of essential oils in markets owing to their multipurpose usage with unique flavour and fragrances. Essential oils are considered as naturally occurring terpenic mixtures [36]. They are a mixture of saturated and unsaturated hydrocarbons, alcohols, aldehydes, esters, ethers, ketones, oxides, phenols and terpenes with a strong characteristic aroma.

Essential oils also called as volatile or ethereal oils are obtained from plants and its secondary metabolites (normally found in special cells or group of cells. They are explored in recent years due to their significant biological activities $[37,38]$. These essential or volatile oil are obtained from various plant parts such as leaves, bud, bark, stem, fruit, roots, twigs, wood, flowers, seeds and herbs. Essential oils are known for its traditional uses such as aromatherapy and flavouring agents in beverages, foods, confectionaries, perfumes, house-hold cleaning products, industrial solvents and cosmetics. The use of essential oils in pharmacies and pharmacopoeias were developed in13th century itself. Essential oils are well known for its anti-bacterial, anti-viral, anti-fungal, anti-helmintic, anti-tumor, anti-oxidant, anti-lice, antidandruff, spasmodic, hormonal and insect repellent activities.

Several of these essential oils are considered as Generally Regarded As Safe (GRAS) Category and are approved by U.S. Food and Drug Administration [39]. Hydro distillation is the common method of producing essential oils. Also, expression, fermentation, enfleurage 
or extraction methods are also used. The method of distillation for essential oils was first adapted before 2000 years ago in India, Egypt and Persia and by $9^{\text {th }}$ century improved by Arabs.

The composition of essential oils depends on the harvesting seasons, geographical sources and type of plant part. Essential oils may contain 20-60 different mixtures, some of them at fairly high concentrations and others at low concentrations. Though, the major components are known to have strong biological activity, minor components also helps in strengthening the effects of the major compounds. Essential oils may contain volatile compounds of terpenoid or non-terpenoid origin, their respective hydrocarbons and oxygenated derivatives, and also nitrogen and sulphur derivatives. The various bioactive compounds include terpenes, terpenoids, mono and sesquiterpenes, molecules with aromatic ring, carbohydrates, phenols, alcohol, ether, aldehydes and ketones. Essential oils with phenols or aldehydes as major component such as cinnamaldehyde, citral, carvacrol, eugenol or thymol are considered more effective as compared to terpene alcohols containing essential oils. Essential oils containing ketones or esters such as $\beta$-myrcene, $\alpha$-thujone or geranyl acetate are known to pose lesser activity. In general, essential oils obtained from herbs harvested during or soon after flowering are known to possess the strongest antimicrobial activity. The enantiomers of the essential oil components exhibit antimicrobial activity to different extent [40].

The importance of various essential oils from natural sources has been explored with great interest owing to its strong medicinal benefits to humankind. The greatest uses of essential oils in European Union are in food as flavourings, perfumes as fragrances and pharmaceuticals for their functional properties. Certain food preservative containing essential oils are commercially available like "DMC Base Natural" that consist of $50 \%$ essential oils (rosemary, sage and citrus) and 50\% glycerol. The antibacterial properties of essential oils are also commercialized as various products including dental root canal sealers, antiseptics, feed supplements for lactating sows and weaned piglets and others. Also, the physiological effects of essential oils are used in commercial potato sprout suppressants and insect repellents. Essential oils from herbs extracted by hexane are prone to exhibit higher antimicrobial activity as compared to steam distilled ones. Thus, essential oils are usually stored in air-tight containers in dark to prevent compositional changes.

Further research on the mechanism of essential oil components on proteins embedded in cytoplasmic membrane and phospholipids has to be pondered for technological applications. The extent to which bacteria can adapt in the presence of essential oils in foods has to be further evaluated. Also, the interaction between essential oils and their components with food ingredients and additives have to be pondered. The role of synergistic effects have to researched to enhance the antimicrobial properties of essential oils, and thereby, to reduce their concentrations. The secondary or the indirect effects on the usage of essential oils have to be explored. The stability of essential oils during food processing has to be investigated as well in depth [41].

\section{Plant Essential Oil Based Nanoemulsions and its Applications}

Research in natural products has captured an important role in the field of food, drug and cosmetic commercial arena. Mostly, essential oils from plants are widely used in pharmaceutical, sanitary, cosmetics, agriculture and food industries for their bactericidal, virucidal, fungicidal, antiparasitical and insecticidal properties. It is also a well-known fact that most of the lipophilic and aromatic bioactive compounds (essential oils and its constituents) pose major disadvantages such as poor aqueous solubility, strong organoleptic characteristics, low stability and limited administration routes. To overcome these drawbacks and to facilitate the applicability of essential oils, several nanocarriers such liposomes, solid lipid nanoparticles, nanoemulsions and polymeric nanoparticles have been extensively studied. These nanocarriers enable controlled release of essential oils, and thereby, have a potential advantage in pharmaceutical dosage forms and have a prolonged preservative effect in cosmetics and foods $[42,43]$. Of these accord, herbal essential oil based nanoemulsions are pondered with great interest in recent years owing to their unique and versatile properties and applications.

\section{Importance}

Though, many new generation antibiotics are being developed, there is still a continuous resistance among many microbial strains against certain antibiotics. Thus, these antibiotics of lipophilic nature are given at higher and multiple dosage concentration. This leads to severe gastrointestinal irritation and other side effects as well. In the context of cancer research, chemotherapy involving the use of highly cytotoxic drugs are been used for several years. This medication could not differentiate between normal and abnormal cells. Hence, by this therapy, normal cells are highly affected by causing sterility, hair cell damage, and others. Moreover, only a limited efficacy of the administered dosage concentration is being reached through the medication.

To bridge this gap, essential oil based nanoemulsions are now gaining great importance in the field of medicine. They improve the aqueous solubility of lipophilic components, enhance oral bioavailability at reduced dosage concentration, provide good stability, transparency and reduce toxic side effects with improved biocompatibility. Of this accord, in recent years, plant essential oil based nanoemulsions are being explored with much importance due to their strong antimicrobial and anticancerous mechanism of action.

The antimicrobial and anticancerous action is additionally enhanced through the co-inclusion of plant essential oils into the nanoemulsion system. Firstly, essential oils, by nature, demonstrate excellent antimicrobial and anticancerous properties. Secondly, nanoemulsions are capable of reducing droplet size diameter to nanometric scale. This causes enhanced surface area for easier interaction with microbial pathogen, and thereby, causes lysis and cell death in the case of antimicrobial activity. In the context of anticancer activity, particle size reduction causes easier permeation of active components through blood vessels without discrimination by natural defence mechanism of host. This enables targeted action and reduces dosage concentration or the multiple dosage regimen with improved efficacy. Thus, plant essential oil based nanoemulsions would capture a valuable role in the modern world of nanomedicine.

\section{Applications}

Antioxidant activity: An ethanolic extract of local Phyllanthus urinaria, incorporated in palm kernel oil based nanoemulsion showed DPPH radical scavenging activity and could be used as a topical delivery for skin anti-ageing too [44]. A transdermal nanoemulsion formulation of cumin essential oil showed effective invitro and invivo antioxidant and hepatoprotective activities [45]. Radix Angelicae sinensis (called as danggui) is a commonly used traditional Chinese medicine with many pharmacological activities. The essential oil of Radix Angelicae sinensis showed concentration-dependent antioxidant activity as investigated by $\mathrm{DPPH}$ radical scavenging assay, ABTS radical scavenging assay, and 
$\beta$-carotene bleaching test., This was mainly attributed to its component, coniferyl ferulate. The antioxidant potential was lower than those of ascorbic acid and BHA [46]. A rice bran oil nanoemulsion protected the stability and antioxidant effect of the propolis extract in the system without interfering its activity as assayed by DPPH scavenging capacity methods [47].

Antimicrobial activity: The antimicrobial activity of nanoemulsions is not specific as that of conventional antibiotics. They exhibit a broadspectrum of antimicrobial activity against bacteria, enveloped viruses and spores at concentrations non-lethal to animals. Their physical kill-on-contact mechanism reduces the emergence of resistance strains. Moreover, they interact with the cell membrane and causes death of the pathogen. A soybean oil based nanoemulsion called BCTP caused $90 \%$ inactivation of Bacillus spores within $4 \mathrm{~h}$. Also, BCTP inhibits biofilm formation of Salmonella spp, E coli 0157:H7, Pseudomonas aeruginosa, and S. aureus. Essential oils of lemongrass, lemon, lemon myrtle, oregano, rosemary, sage, thyme, clove and tea tree are known to exhibit antimicrobial activity, but excess amounts might affect organileptic property as well [48]. A nanoemulsion of Thymus daenensis essential oil produced by high intensity ultrasound technique demonstrates superior antibacterial activity with increased ability to disrupt cell membrane integrity of a food-borne pathogen, $E$. coli [49]. Nanoemulsion of eucalyptus oil prepared by ultrasonication was impregnated with chitosan film showed enhanced antibacterial activity against Staphylococcus aureus and also could help in wound management studies [50]. Also, eucalyptus oil nanoemulsion showed potent antibacterial activity against Proteus mirabilis. Thus, a topical formulation of this product could be effective against uropathogens [51]. Nanoemulsions of peppermint essential oil showed prolonged antibacterial activity when compared with oil alone. Nanoemulsions of lemongrass essential oil prepared by microfluidization technique demonstrated faster inactivation kinetics in E. coli as compared to their sonicated nanoemulsions [52]. Melaleuca alternifolia essential oil based nanoemulsion showed lower minimum inhibitory concentration against Saccharomyces cerevisiae, Escherichia coli, and Lactobacillus delbrueckii. This, in combination with fruit juices, the shelf life could be extended. Also, Similarly, Basil oil (Ocimum basilicum) nanoemulsion showed dose-dependent effect over E. coli viability [53]. Neem oil nanoemulsion prepared by ultrasonication technique causes disruption of cell membrane integrity of the bacterial pathogen Vibrio vulnificus. Moreover, this nanoemulsion was found to be non-toxic to human lymphocytes at lower concentrations, whereas, it induced toxicity at higher concentrations [54]. The eucalyptus oil nanoemulsion prepared by ultrasonication technique with a mean droplet diameter of 3.8 $\mathrm{nm}$ showed complete loss of viability of $S$. aureus within 15 minutes of interaction as assayed by time kill analysis. This nanoemulsion when tested on Wistar rats showed higher wound healing potential as compared to controls and neomycin treated ones with no skin irritation, and thus could be used as topical applications as well [55].

Various antimicrobial assays may include growth kinetics, plate count method, reactive oxygen species, cellular uptake, lactate dehydrogenase assays and micoscopis analysis.

Anticancer activity: Over six millions of years, it is claimed that cancer is the second major cause of death worldwide. Recent research on natural products has increased to improve the current treatment in patients with multidrug resistance. These scientific studies mostly on plants have led to the development of many drugs including taxol, camptothecin, vincristine and vinblastine. There are many publication reports on the anticancer activity of essential oils. Of this accord, the first ever publication dated 1960s. As of now, essential oils are investigated on glioblastoma, melanoma, leukemia and various cancers of oral, bone, breast, colon, cervix, kidney, liver, lung, ovary, pancreas, prostate and uterus cancers. In the recent decade, about hundred essential oils isolated from more than twenty plant families have been tested for more than twenty types of cancers.

The in vivo antiangiogenic effect of Betulin (a secondary metabolite of plants abundantly und in the outer bark of birch tree Betulaceae sp.) was reported in the chorioallantoic membrane (CAM) model of chicken embryos. Betulin inhibits the formation of new capillaries by targeting the endothelial cells. This activity was further enhanced using nanoemulsion formulation of Betulin. This increases the penetrability to extra embryonic tissues. Betulin also acts as antimutagenic agent of skin carcinogenesis. The topical formulation of Betulin nanoemulsion tested on C57BL/6J type mouse skin demonstrated reduced skin lesions and irritation with decreased erythema [56]. Nanoemulsions of pine nut oil was used in the encapsulation of paclitaxel (PTX), apoptotic signaling molecule C-(6)-ceramide (CER) and combinations of both PTX and CER. It was demonstrated that the combinational effects of nanoemulsion encapsulated PTX and CER showed higher cytotoxic effects in brain tumor cells as compared to administration of individual agents. Thus, PTX and CER could augment therapeutic activity in glioblastoma [57].

The anticancer mechanistic study is carried out by cytotoxic assays (MTT, Resazurin/Alamar Blue dye, Annexin V-FITC, Western Blot, GSH/GSSG assay, DCFH-DA assay and others), genotoxic assay (Comet assay) and microscopic (fluorescence microscopy) analysis.

Insecticidal and larvicidal activity: The plant based repellents are used traditionally to protect against host-seeking mosquitoes. Citronella oil nanoemulsion shows decrease in release rates and thereby, enhances the mosquito protection rates. Also, the microencapsulation using gelatin-arabic gum microcapsules prolongs the natural repellence of citronella. The use of these novel technologies may fasten the use of plant oil based natural repellents in markets [58]. Hairy basil oil and vetiver oil nanoemulsions prolonged the repellent activity against Aedes aegypti.

Pterodon emarginatus Vogel is a Brazilian species of the family Fabaceae. It demonstrated potent larvicidal activity against Aedes aegypti. The authors developed P. emarginatus oil based nanoemulsion to control dengue by possibly suspending larvicidal agent in water to make the active lipophilic natural product available. The possible mode of action involves reversible inhibition of acetyl cholinesterase and the formulated nanoemulsion was found non- toxic for mammals [59].

Nanoemulsions of neem oil nanoemulsion enhanced the bioavailability and pesticidal action [60]. A seed and leaf extract of eucalyptus oil nanoemulsion was produced into pellets and this showed fumigant toxicity against five important pests such as Oryzaephilus surinamensis, Sitophilus oryzae, Tribolium confusum, Callosobruchus maculatus and Rhyzopertha dominica [61]. A clove oil nanoemulsion stable for about six months was developed by ultrasound technique as a green pesticide [62]. Formulations with high eucalyptol content were effective against red flour beetle (Tribolium castaneum). However, their effects decreased after two months. To enhance their insecticidal effects, the formulations were further combined with the aqueous extract of Jatropha curcas and Pongamia glabra. Also, trypanocidal activity of andiroba (Carapa guianensis) and aroeira (Schinus molle) essential oils associated to nanoemulsions were also studied. 
Food industry: In food industries, the formation of nanoemulsions using triacylglycerols oils like corn, soybean, sunflower, safflower, olive, flaxseed, algae or fish oils is a better owing to its low cost and abundance. Most of these oils mainly consist of long-chain triacylglycerols and thus could be used in food applications. The formation of nanoemulsions using the medium-chain and short-chain triacylglycerols present in these oils could be challenging due to their low polarity, high interfacial tension and high viscosity. An orange oil nanoemulsion prepared by ultrasonication technique showed antiyeast activity against Saccharomyces cerevisiae. Their activity was also evaluated in apple juices that showed complete loss of viability [63]. There is a report indicating an increase in bioaccessibility of carotenoid from yellow peppers when consumed with corn oil nanoemulsions as excipients. Also, there was an increase in in-vitro bioaccessibility of powdered curcumin after mixing with its excipient nanoemulsions. This showed a higher transfer of curcumin powder to oil droplets when the nanoemulsion was incubated at $100 \otimes \mathrm{C}[64]$. The effects on ultrasound processing of lemon grass oil-alginate nanoemulsions is studied as it could incorporate this essential oil into the food matrix and enhance its bioavailability [65]. An oregano oil nanoemulsion tested on fresh lettuce shows effective antimicrobial activity against food borne pathogens such as Listeria monocytogenes, Salmonella Typhimurium and Escherichia coli O157:H7. Cavacrol nanoemulsion shows effectiveness against Salmonella enterica Enteritidis and E. coli on radish seeds, mung bean and alfalfa seeds and not on broccoli seeds. A lemongrass essential oil nanoemulsion tested on plums shows effectiveness against Salmonella and E. coli. A chitosan coated mandarin essential oil nanoemulsion treated on green beans is tested against Listeria innocua that shows promising applications as food products. The essential oil based nanoemulsions have showed effective control of food borne pathogens on food. Yet, there are very limited studies on food models. Thus, there is a growing challenge in the food industries to develop consumer confidence to use nano food ingredients [66].

Cosmetics: Phytocompounds are used for decades for their potential beauty applications such as sunscreen, moisturizing, anti-aging and skin-based therapy. The nano based delivery technologies are currently used for sustained and improved delivery of phytocompounds and to overcome the low permeation and instability problems of these bioactive compounds. Nanoemulsions of plant based bioactive compounds such as flavonoids and polyphenols improve the cosmeceutical value due to very slight toxicity. Quercetin rich nanoemulsions increased skin retention of bioactive components with improved antioxidant activities in porcine skin. A multiple nanoemulsion of genistein coloaded with tocomin offers skin protection from UV, and could be used as sunscreen lotion. Curcumin nanoemulsion with improved transdermal availability could be used in skin care sectors. Nanoemulsions of Achyrocline extracts demonstrated protection against herpes virus infections. Nanoemulsions of Aloe vera extracts showed skin rejuvenation and anti-wrinkle activity. A rice bran oil nanoemulsion could be used as moisturizers with anti-ageing activity. Nanoemulsions of D-Limonene and tocopherol were used in skin care applications. Vitamin E enriched palm oil nanoemulsions showed improved stability and be used for further applications. Genistein loaded nanoemulsion enhances the delivery of isoflavones to skin with good skin protecting activity. A pomegranate seed oil nanoemulsion demonstrated increased skin protection against photo damage with improved antioxidant activities [67].

Drug delivery systems: Nanoemulsions plays an important role in pharmacy as drug delivery systems owing to their solubilization capacity of non-polar compounds and facilitates administration through various routes. Also, nanoemulsions could serve as the ideal delivery platform for the delivery of phyto active compounds as it shows improved absorption, permeation, and stability of certain herbal based drugs [68]. Clove and cinnamon essential oils were used in encapsulation of three contemporary drugs including ramipril (antihypertensive), azithromycin (antibacterial) and fluconazole (antifungal) drugs respectively. These oils showed higher solubilization of these drugs. So, a nano-scaled drug delivery system was prepared and characterized using simple titration microemulsion technique and ultrasonication technique. Both the antibacterial and antifungal drugs showed a higher efficacy against $S$. aureus and C. albicans strains respectively due to the combinational effects of the drug and the co-inclusion of essential oil into the system. Moreover, this could reduce the dosage concentrations of the conventional drugs used [69].

Future prospectives: Spices are used in most food preparations and they are known to possess strong medicinal values as well. Spices are also considered as an important heritage of India as about 63 different varieties of spices are grown in India. The active ingredients and essential oils obtained from spices have demonstrated excellent antibacterial, antifungal, anticancer, and other activities. But the efficacies of these spice essential oils and their bioactive ingredients are not completely achieved due to the lipophilic nature and poor aqueous solubility [7073].

The advent of Nanoscience and Nanotechnology has aroused a greater expectation among pharmaceutical scientists to overcome these drawbacks. Moreover, nanoemulsions are known to have a strong impact towards antimicrobial and anticancer therapy. They exhibit wide spectrum antimicrobial action against bacteria, fungi and viruses. They demonstrate an excellent theranostic platform for image guided therapy. Also, they serve as excellent nanocarriers of many anticancer compounds for easy permeation through small capillaries of blood vessels. So currently, researchers have pondered on the development of spice based nanoemulsion systems to enhance their solubility and to improve the efficacy of their activities as potential antimicrobial and anticancer agents. Moreover, this spice based nanoemulsions could pose reduced toxic effects, biocompatibility and bioavailability as compared to other conventional antibiotic treatment and chemotherapy. Though, literatures are very limited, there is strong evidence concerning the improved antimicrobial and anticancer mechanistic activities of the spice based nanoemulsions [74-76].

\section{Conclusion}

Though, there are many reports on various essential oil based nano formulations of medical importance [77], there are only limited literatures concerning spice (oil and active ingredients) based nanoemulsions. Curcumin incorporated polymeric nanocapsules formed by ultrasound was optimized and characterized for lipophilic delivery of food active ingredients [78]. A high shear homogenization technique using microfluidizer was used in the formulation of various essential oil based nanoemulsions, of which, few were spice oil based. This includes lemongrass, clove, tea tree, thyme, geranium, marjoram, palmarosa, rosewood, sage or mint nanoemulsions respectively. Of these essential oil based nanoemulsions, lemongrss, clove, thyme, and palmarosa demonstrated superior bactericidal activity against Escherichia coli. Moreover, lemongrass and clove emulsions demonstrated higher inactivation kinetics [79]. An investigation on the infusion of carvacrol into food matrices revealed particle size reduction to nanometric range caused an increased diffusivity with higher antimicrobial action [80]. Eugenol incorporated nanoemulsions 
Citation: Nirmala MJ, Nagarajan R (2017) Recent Research Trends in Fabrication and Applications of Plant Essential Oil Based Nanoemulsions. J Nanomed Nanotechnol 8: 434. doi: 10.4172/2157-7439.1000434

demonstrated the apoptosis of colon (HTB37) and liver (HB8065) cancer cells through the generation of reactive oxygen species [81]. Cavacrol essential oil based nanoemulsion system was produced by spontaneous emulsification method. This produced stable nanoemulsion with antimicrobial property [82]. A nanoemulsion was also prepared using ginger extract and optimized through various process parameters for enhanced stability of 6-gingerol [83]. Spice (Drimys angustifolia and Drimys brasiliensis) essential oil based nanoemulsions were prepared to demonstrate their potent cytotoxic effects. Among these, D. brasiliensis showed reduced cell viability of human glioblastoma (U$138 \mathrm{MG}$ ) and human bladder carcinoma (T24) cell lines and caused late apoptosis. A high pressure homogenization technique was involved in the incorporation of carvacrol, limonene and cinnamaldehyde into sunflower oil nanoemulsion systems. These demonstrated significant antimicrobial activity against Lactobacillus delbrueckii and Saccharomyces cerevisiae [84]. Cavacrol and eugenol nanoemulsions prepared by high pressure homogenizer or ultrasonicator showed improved antimicrobial activity against Escherichia coli and Listeria innocua [85]. Turmeric nanoemulsions prepared by high energy methods demonstrated antibacterial activity against Staphylococcus aureus [72]. An ultrasonic emulsification technique was involved in preparing cinnamon oil nanoemulsion system. The antimicrobial mechanism of this spice oil based nanoemulsion against Bacillus cereus was studied in detail [86]. Eugenol incorporated nanoemulsion demonstrated improved potency against Staphylococcus aureus and also significantly reduced the bacterial population in orange juice [87]. A recent finding indicates that Nigella sativa essential oil based nanoemulsion prepared by ultrasonication technique has potent application in breast cancer therapy [88]. Also, few more reports on the existence of microemulsions of carvacrol, eugenol, clove, corn, peppermint, orange, cotton seed and cinnamon oils respectively [8993].

The combinational effects of spices and nanoemulsions would demonstrate enhanced efficacy to a greater extent. Moreover, this could serve as an effective drug delivery system with targeted site of action. This could meet the patient's compliance in a better way. Thus, this scientific area of research based on spice based nanoemulsions to improve antimicrobial and anticancer efficacy would pose a major impact in the field of Nanomedicine, as it could be used as an alternative to standard/conventional antibiotic therapy and chemotherapy by minimizing dosage concentration and by limiting multiple doses.

\section{Acknowledgement}

The authors thank DST-SERB (Young Scientist Scheme), Government of India for all sources of support and funding in this research area.

\section{References}

1. International Union of Pure and Applied Chemistry Manual of Colloid Science. London: Butterworth; 1972.

2. Tadros TF (2013) Emulsion formation, stability and rheology. In: Tadros TF (ed.) Emulsion formation and stability First Edition. Wiley-VCH Verlag $\mathrm{GmbH}$ and Co. KGaA.

3. Achouri A, Zamani Y, Boye JI (2012) Stability and physical properties of emulsions prepared with and without soy proteins. Journal of Food Research 1: 254-267.

4. Usaid ASA, Premkumar J, Ranganathan TV (2014) Emulsion and it's applications in food processing -a review. Int Journal of Engineering Research and Applications 4: 241-248.

5. Barnes HA (2004) Chapter 18: Rheology of emulsions. In: Petsev DN (ed.) Emulsions: Structure stability and interactions - Interface Science and Technology. Elsevier Academic Press.
6. Madaan V, Chanana A, Kataria MK, Bilandi A (2014) Emulsion technology and recent trends in emulsion applications. Int Res J Pharm 5: 533-542.

7. Garg J, Kumar P (2014) Emerging trends in nanoemulsions design and therapeutics -a review. Asian Journal of Pharmaceutical Sciences and Clinical Research 2: 1-16.

8. Gupta A, Eral HB, Hattona TA, Doyle PS (2016) Nanoemulsions: formation, properties and applications. Soft Matter 12: 2826-2841.

9. Anton N, Vandamme $T$ (2009) The universality of low-energy nanoemulsification. International Journal Pharmaceutics 377: 142-147.

10. Tadros T, Izquierdo P, Esquena J, Solans C (2004) Formation and stability of nanoemulsions. Advances in Colloids and Interface Science 108-109: 303-318.

11. McClements D (2005) Food emulsions: principles, practices, and techniques CRC Press, Boca Raton, FL.

12. Anton N, Gayet JP, Benoit P, Saulnier P (2007) Nano-emulsions and nanocapsules by the pit method: an investigation on the role of the temperature cycling on the emulsion phase inversion. International Journal of Pharmaceutics 344: 44-52.

13. Kralova I, Sjoblom J (2009) Surfactants used in food industry: a review. Journal of Dispersion Science and Technology 30: 1363-1383.

14. Wooster T, Golding M, Sanguansri $P$ (2008) Impact of oil type on nanoemulsion formation and Ostwald ripening stability. Langmuir 24: 12758-12765.

15. McClements DJ, Rao J (2011) Food-grade nanoemulsions: formulation, fabrication, properties, performance, biological fate, and potential toxicity. Critical Reviews in Food Science and Nutrition 51: 285-330.

16. Lovelyn C, Attama AA (2011) Current state of nanoemulsions in drug delivery Journal of Biomaterials and Nanobiotechnology 2: 626-639.

17. Mason TG, Graves SM, Wilking JN, Lin MY (2006) Extreme emulsification: formation and structure of nanoemulsions. Journal of Physics and Condensed Matter 9: 193-199.

18. Jafari SM, He Y, Bhandari B (2007) Optimization of nanoemulsion production by microfluidization. European Food Research Technology 225: 733-741.

19. Quin C, Mc Clement DJ (2011) Formation of nanoemulsions stabilized by model food grade emulsifiers using high pressure homogenization: factors effecting particle size. Food Hydrocolloids 25: 1000-1008.

20. Sonneville-Aubrun O, Simonnet JT, L'Alloret F (2004) Nanoemulsions: a new vehicle for skincare products. Advances in Colloids and Interface Science 108109: 145-149.

21. Solans C, Izquierdo P, Nolla J, Azemar N, Garcia-Celma MJ (2005) Nanoemulsions Current Opinion in Colloid and Interface Science. 10: 102-110.

22. Sole I, Pey CM, Maestro A, Gonzalez C, Porras M, et al. (2010) Nanoemulsions prepared by phase inversion composition method: preparation variables and scale up. Journal of Colloid and Interface Science 344: 417-423.

23. Bainun IN, Hashimah AN, Syed-Hassan SSA (2015) Nanoemulsion: formation characterization, properties and applications - a review. Advanced Materials Research 1113: 147-152.

24. Shafiq S, Faiyaz S, Sushma T, Ahmad FJ, Khar RK, et al. (2007) Development and bioavailability assessment of ramipril nanoemulsion formulation. European Journal of Pharmaceutics and Biopharmaceutics 66: 227-243.

25. Amselem S, Friedman D (1998) Submicron emulsions as drug carriers fo topical administration. In: Benita S (ed.) Submicron emulsions in drug targeting and delivery. The Netherlands, Harwood Academic Publishers, Amsterdam 153-173.

26. Shakeel F, Baboota S, Ahuja A, Ali J, Faisal MS, et al. (2008) Stability evaluation of Celecoxib nanoemulSion containing tween 80 . Thailand Journal of Pharmaceutical Science 32: 4-9.

27. Shakeel F, Baboota S, Ahuja A, Ali J, Shafiq S (2008) Skin permeation mechanism of Aceclofenac using novel nanoemulsion formulation. Pharmazie 63: $580-584$.

28. Baboota S, Shakeel F, Ahuja A, Ali J, Shafiq S (2007) Design development and evaluation of novel nanoemulsions formulations for transdermal potential of Celecoxib. Acta Pharmaceutica 8: 316-332.

29. Chime SA, Kenechukwu FC, Attama AA (2014) Nanoemulsions - advances in formulation, characterization and applications in drug delivery. In: Sezer AD 
Citation: Nirmala MJ, Nagarajan R (2017) Recent Research Trends in Fabrication and Applications of Plant Essential Oil Based Nanoemulsions. J Nanomed Nanotechnol 8: 434. doi: 10.4172/2157-7439.1000434

Page 9 of 10

(ed.) Application of nanotechnology in drug delivery. InTech.

30. Patel RP, Joshi JR (2012) An overview on nanoemulsion: a novel approach. International Journal of Pharmaceutical Sciences and Research 3: 4640-4650.

31. Gutiérrez JM, González C, Maestro A, Solè I, Pey CM, et al. (2008) Nanoemulsions: New applications and optimization of their preparation. Current Opinion in Colloid and Interface Science 13: 245-251.

32. Kumar GP, Divya A (2015) Nanoemulsion based targeting in cancer therapeutics. Med Chem 5: 272-284.

33. Liu L, Bagia C, Janjic JM (2015) The first scale-up production of theranostic nanoemulsions. BioResearch Open Access 4.1: 218-228.

34. Maali A, Mosavian MTH (2013) Preparation and application of nanoemulsions in the last decade (2000-2010). Journal of Dispersion Science and Technology 34: 92-105.

35. Mehta RN, More U, Malek N, Chakraborty M, Parikh PA (2015) Study of stability and thermodynamic properties of water-in-diesel nanoemulsion fuels with nano-Al additive. Appl Nanosci 5: 891-900.

36. Daferera DJ, Ziogas BN, Polissiou MG (2003) The effectiveness of plant essential oils on the growth of Botrytis cinerea, Fusarium sp. and Clavibacter michiganensis subsp. Michiganensis. Crop Protection 22: 39-44.

37. Seow YX, Yeo CR, Chung HL, Yuk HG (2014) Plant essential oils as active antimicrobial agents. Crit Rev Food Sci Nutr 54: 625-44.

38. Vivek K. Bajpai, Kwang-Hyun Baek (2016) Biological Efficacy and Application of Essential Oils in Foods-A Review. Journal of Essential Oil Bearing Plants 19: 1-19.

39. Ali B, Al-Wabel NA, Shams S, Ahamad A, Khan SA, et al. (2015) Essentia oils used in aromatherapy: a systemic review. Asian Pacific Journal of Tropical Biomedicine 5: 601-611.

40. Perricone M, Arace E, Corbo MR, Sinigaglia M, Bevilacqua A (2015) Bioactivity of essential oils: a review on their interaction with food components. Frontiers in Microbiology 6: 1-7.

41. Burt S (2004) Essential oils: their antibacterial properties and potentia applications in foods-a review. International Journal of Food Microbiology 94 : 223-253.

42. São Pedro1 A, Espirito Santo I, Silva CV, Detoni C, et al. (2013) The use of nanotechnology as an approach for essential oil-based formulations with antimicrobial activity. Microbial pathogens and strategies for combating them: science, technology and education (A. Méndez-Vilas, Ed.). Formatex Research Center 2: 1364-1374.

43. Bayala B, Bassole IHN, Scifo R, Gnoula C, More L, et al. (2014) Anticancer activity of essential oils and their chemical components - a review. Am J Cancer Res 4: 591-607.

44. Mahdi ES, Noor AM, Sakeena MH, Abdullah GZ, Abdulkarim MF, et al. (2011) Formulation and in vitro release evaluation of newly synthesized palmkerne oil esters-based nanoemulsion delivery system for $30 \%$ ethanolic dried extract derived from local Phyllanthus urinaria for skin antiaging. Int $\mathrm{J}$ Nanomed 6: 2499-2512.

45. Mostafa DM, Kassem AA, Asfour MH, Al Okbi SY, Mohamed DA, et al. (2015) Transdermal cumin essential oil nanoemulsions with potent antioxidant and hepatoprotective activities: In-vitro and in-vivo evaluation. Journal of Molecular Liquids 212: 6-15.

46. Su-Ying Li, Yue Yu, Shao-Ping Li (2007) Identification of Antioxidants in Essential Oil of Radix Angelicae Sinensis Using HPLC Coupled with DAD-MS and ABTS-Based Assay. J Agric Food Chem 55: 3358-3362.

47. Mauludin R, Fidrianny I, Primaviri DS, Alifiana O, Mukti RR (2015) Natural Product Utilization As Antioxidant Formulated in Nanoemulsion. Research and Development on Nanotechnology in Indonesia (RDNI) 2: 20-27.

48. Shams N, Sahari MA (2016) Nanoemulsions: Preparation, Structure, Functional Properties and their Antimicrobial Effects. Appl Food Biotechnol 3: 38-149.

49. Moghimi R, Ghaderi L, Rafati H, Aliahmadi A, McClements DJ (2016) Superior antibacterial activity of nanoemulsion of Thymus daenensis essential oil against E. coli. Food Chemistry 194: 410-415.

50. Sugumar S, Mukherjee A, Chandrasekaran N (2015) Eucalyptus oil nanoemulsion-impregnated chitosan film: antibacterial effects against a clinical pathogen, Staphylococcus aureus, in vitro. International Journal of
Nanomedicine 10: 67-75

51. Saranya S, Chandrasekaran N, Mukherjee A (2012) Antibacterial activity of eucalyptus oil nanoemulsion against Proteus mirabilis. Int J Pharm Pharm Sc 4: 668-671.

52. Odriozola-Serrano I, Oms-Oliu G, Martín-Belloso O (2014) Nanoemulsionbased delivery systems to improve functionality of lipophilic components. Front Nutr 1: 24.

53. Zorzi GK, Carvalho ELS, von Poser GL, Teixeira HF (2015) On the use of nanotechnology-based strategies for association of complex matrices from plant extracts. Revista Brasileira de Farmacognosia 25: 426-436.

54. Jerobin J, Makwana P, Suresh Kumar RS, Sundaramoorthy R, Mukherjee A, et al. (2015) Antibacterial activity of neem nanoemulsion and its toxicity assessment on human lymphocytes in vitro. International Journal of Nanomedicine 10: 77-86.

55. Sugumar S, Ghosh V, Nirmala MJ, Mukherjee A, Chandrasekaran N (2014) Ultrasonic emulsification of eucalyptus oil nanoemulsion: antibacterial activity against Staphylococcus aureus and wound healing activity in Wistar rats. Ultrason Sonochem 21: 1044-9.

56. Król SK, Kielbus M, Rivero-Müller A, Stepulak A (2015) Comprehensive Review on Betulin as a Potent Anticancer Agent. BioMed Research International 1: 11

57. Desai A, Vyas T, Amiji M (2008) Cytotoxicity and apoptosis enhancement in brain tumor cells upon coadministration of paclitaxel and ceramide in nanoemulsion formulations. J Pharm Sci 97: 2745-56.

58. Maia MF, Moore SJ (2011) Plant-based insect repellents: a review of their efficacy, development and testing. Malaria Journal S11: 1-15.

59. Oliveira AEMFM, Duarte JL, Amado JRR, Cruz RAS, Rocha CF, et al. (2016) Development of a Larvicidal Nanoemulsion with Pterodon emarginatus Voge Oil. PLoS ONE 11: e0145835.

60. Sagiri SS, Anis A, Pal K (2016) Review on Encapsulation of Vegetable Oils: Strategies, Preparation Methods, and Applications. Polymer-Plastics Technology and Engineering 55: 291-311.

61. Zibaee I, Farahani HM, Razi JM, Moghaddam MN (2016) Comparison of nanopellets formulation with phostoxin against five important pests of stored products. Journal of Entomology and Zoology Studies 4: 80-85.

62. Evaluation of critical parameters for preparation of stable clove oil nanoemulsion. Mohammad Hassan Shahavi, Morteza Hosseini, Mohsen Jahanshahi, Rikke Louise Meyer, Ghasem Najafpour Darzi. Arabian Journal of Chemistry. In Press.

63. Sugumar S, Singh S, Mukherjee A, Chandrasekaran N (2016) Nanoemulsion of orange oil with non ionic surfactant produced emulsion using ultrasonication technique: evaluating against food spoilage yeast. Appl Nanosci 6: 113-120.

64. Salvia-Trujillo L, Martín-Belloso O, McClements DJ (2016) Excipient Nanoemulsions for Improving Oral Bioavailability of Bioactives. Nanomaterials 6: 17 .

65. Olorunnisola SK, Asiyanbi HT, Hammed AM, Simsek S (2014) Biological properties of lemongrass: An overview. IFRJ 21: 455-462.

66. Amaral DMF, Bhargava K (2015) Essential oil nanoemulsions and food applications. Adv Food Technol Nutr Sci Open 1: 84-87.

67. Ganesan P, Choi DK (2016) Current application of phytocompound-based nanocosmeceuticals for beauty and skin therapy. International Journal of Nanomedicine 11: 1987-2007.

68. Patil PA, Bhutkar BR (2016) Biomedical Application of Nanoemulsion- A Features Review. International Journal of Research Methodology 1: 37-58.

69. Nirmala MJ, Mukherjee A, Chandrasekaran N (2014) A distinctive drug delivery system focused on contemporary drugs. Adv Sci Eng Med 6: 719-735.

70. Skrinjar MM, Nemet NT (2009) Antimicrobial effects of spices and herbs essential oils. Acta peridica technologica 40: 195-209.

71. Singh S, Majumdar DK (1999) Evaluation of the gastric antiulcer activity of fixed oil-Ocimum sanctum (Holy Basil). Journal of Ethnopharmacology 65: 6513-6519.

72. Sarojini S, Sundar K, Anjali CH, Ravindran A (2014) Formation and stability of oil-in-water nanoemulsion containing turmeric oil. Biotechnology: An Indian Journal 9: 171-175. 
Citation: Nirmala MJ, Nagarajan R (2017) Recent Research Trends in Fabrication and Applications of Plant Essential Oil Based Nanoemulsions. J Nanomed Nanotechnol 8: 434. doi: 10.4172/2157-7439.1000434

73. Gomes MRF, Schuh RS, Jacques ALB, Augustin OA, Bordignon SAL, et al. (2013) Citotoxic activity evaluation of essential oils and nanoemulsions of Drimys angustifolia and D. brasiliensis on human glioblastoma (U-138 MG) and human bladder carcinoma (T24) cell lines in vitro. Brazilian Journal of Pharmacognosy 23: 259-267.

74. Hwang Y, Ramalingam K, Bienek DR, Lee V, You T, et al. (2013) Antimicrobial activity of nanoemulsion in combination with cetylpyridinium chloride in multi-drug resistant in Acinetobacter baumannii. Antimicrobial Agents and Chemotherapy 54: 3568-3575.

75. Gianella A, Jarzyna PA, Mani Y, Ramachandran S, Calcagno C, et al. (2011) A multifunctional nanoemulsion platform for imaging guided therapy evaluated in experimental cancer. ACS Nano 5: 4422-4433.

76. Anchapreeda S, Fukumori Y, Okonogi S, Ichikawa H (2012) Preparation of lipid nanoemulsions incorporating curcumin for cancer therapy. Journal of Nanotechnology.

77. Bilia AR, Guccione C, Isacchi B, Righeschi C, Firenzuoli F, et al. (2014) Essential oils loaded in nanosystems: a developing strategy for a successful therapeutic approach. Evidence-based Complementary and Alternative Medicine.

78. Abbas S, Karangwa E, Bashari M, Hayat K, Hong X, et al. (2015) Fabrication of polymeric nanocapsules from curcumin-loaded nanoemulsion templates by self-assembly. Ultrasonic Sonochemistry 23: 81-92.

79. Salvia-Trujillo L, Rojas-Grau A, Soliva-Fortuny R, Martin-Belloso O (2015) Physicochemical characterization and antimicrobial activity of food-grade emulsions and nanoemulsions incorporating essential oils. Food Hydrocolloids 43: $547-556$

80. Donsi F, Cuomo A, Marchese E, Ferrari G (2014) Infusion of essential oils for food stabilization: unravelling the role of nanoemulsion-based delivery systems on mass transfer and antimicrobial activity. Innovative Food and Emerging Technologies 22: 212-220.

81. Majeed H, Antoniou J, Fang Z (2014) Apoptotic effects of eugenol-loaded nanoemulsions in human colon and liver cancer cell lines. Asian Pacific Journal of Cancer Prevention 15: 9159-9164.

82. Chang Y, McLandsborough L, McClements DJ (2013) Physicochemical properties and antimicrobial efficacy of carvacrol nanoemulsions formed by spontaneous emulsification. Journal of Agricultural and Food Chemistry 61: 8906-8913.
83. Uthumpa C Indranupakorn R, Asasutjarit R (2013) Development of nanoemulsion formulations of ginger extract. Advanced Material Research 684: 12-15.

84. Donsi F, Annunziata M, Vincensi M, Ferrari G (2012) Design of nanoemulsionbased delivery systems of natural antimicrobials: effect of the emulsifier. Journal of Biotechnology 159: 342-350.

85. Terjung N, Loffler M, Gibis M, Hinrichs J, Weiss J (2012) Influence of droplet size on the efficacy of oil-in-water emulsions loaded with phenolic antimicrobials. Food and Function 3: 290-301.

86. Ghosh V, Saranya S, Mukherjee A, Chandrasekaran N (2013) Cinnamon oi nanoemulsion formulation by ultrasonic emulsification: investigation of its bactericidal activity. Journal of Nanoscience and Nanotechnology 13: 114-122.

87. Ghosh V, Mukherjee A, Chandrasekaran N (2014) Eugenol-loaded antimicrobia nanoemulsion preserves fruit juice against microbial spoilage. Colloids and Surfaces B Biointerfaces 114: 392-397.

88. Periasamy VS, Athinarayanan J, Alshatwi AA (2016) Anti-cancer activity of an ultrasonic nanoemulsion formulation of Nigella sativa L. essential oil on human breast cancer cells. Ultrasonic Sonochemistry 31: 449-455

89. Gaysinsky S, Taylor TM, Davidson PM, Bruce BD, Weiss J (2007) Antimicrobia efficacy of eugenol microemulsions in milk against Listeria monocytogenes and Escherichia coli 0157:H7. Journal of Food Protection 70: 2631-2637.

90. Gaysinsky S, Davidson PM, McClements DJ, Weiss J (2008) Formulation and characterization of phytophenol-carrying antimicrobial microemulsions. Food Biophysics 3: 54-65

91. Hamed SF, Sadek Z, Edris A (2012) Antioxidant and antimicrobial activities of clove bud essential oil and eugenol nanoparticles in alcohol-free microemulsion. Journal of OleoScience 61: 641-648.

92. Gupta S, Sanyal SK, Datta S, Moulik SP (2006) Preparation of prospective plant oil derived micro-emulsion vehicles for drug delivery. Indian Journal of Biochemistry and Biophysics 43: 254-257.

93. Ghosh V, Saranya S, Mukherjee A, Chandrasekaran N (2013) Antibacteria microemulsion prevents sepsis and triggers healing of wound in Wistar rats. Colloids and Surfaces B: Biointerfaces 105: 152-157. 